\title{
UCRL-JRNL-204491
}

LAWRENCE LIVERMORE NATIONAL LABORATORY

\section{Repair of Phase Defects in Extreme-Ultraviolet Lithography Mask Blanks}

S.P. Hau-Riege, A.Barty, P.B. Mirkarimi, S.Baker, M.A. Coy, M.Mita, V.E. Robertson, T.Liang, and A.Stivers

June 4, 2004

Journal of Applied Physics 
This document was prepared as an account of work sponsored by an agency of the United States Government. Neither the United States Government nor the University of California nor any of their employees, makes any warranty, express or implied, or assumes any legal liability or responsibility for the accuracy, completeness, or usefulness of any information, apparatus, product, or process disclosed, or represents that its use would not infringe privately owned rights. Reference herein to any specific commercial product, process, or service by trade name, trademark, manufacturer, or otherwise, does not necessarily constitute or imply its endorsement, recommendation, or favoring by the United States Government or the University of California. The views and opinions of authors expressed herein do not necessarily state or reflect those of the United States Government or the University of California, and shall not be used for advertising or product endorsement purposes. 


\title{
Repair of phase defects in extreme-ultraviolet lithography mask blanks
}

\author{
Stefan P. Hau-Riege, Anton Barty, Paul B. Mirkarimi, and Sherry Baker \\ Lawrence Livermore National Laboratory, Livermore, CA 94550
}

Michael A. Coy, Masaaki Mita, and Vernon E. Robertson

JEOL USA, Inc, Peabody, MA 01960

\author{
Ted Liang and Alan Stivers \\ Intel Corporation, Santa Clara, CA 95052
}

Phase defects that introduce errors in the printed image are one of the major yield limiters for reticles in extreme ultraviolet lithography. The basis for a reticle is a mask blank, consisting of an ultra-low-expansion substrate and a reflective multilayer stack that is made up of Mo and Si. A potential methodology for repairing phase defects in these mask blanks is to locally irradiate it with a high-resolution electron beam to induce structural deformations that cancel out the distortion of the multilayer. We present experimental and modeling results that show that the interaction of an electron beam with a mask blank can only be understood when the contraction of the multilayer through silicide formation and substrate compaction, as well as the mechanical response of the mask blank, are considered. One of the consequences is that electron beams with energies around $10 \mathrm{keV}$ that are less than $20 \mathrm{~nm}$ in diameter induce depressions in 
multilayers made up of $50 \mathrm{Mo} / \mathrm{Si}$ bilayers that have a surprisingly large diameter of about a micrometer. Our models suggest that these unacceptably large diameters can only be reduced if the compaction of the substrate is prevented. 


\section{Introduction}

Extreme ultraviolet lithography (EUVL) is one of the leading candidates for the $32 \mathrm{~nm}$ technology node for semiconductor integrated circuits. EUVL incorporates the use of allreflective optics, including reflective masks. The basis for an EUVL reticle is a mask blank that consist of an ultra-low-expansion substrate and a multilayer stack that is made up of typically 50 bilayers of Mo and Si. Si acts primarily as the low-absorption spacer material, and Mo as the absorber. In this multilayer stack, a standing wave field forms to reflect light at a wavelength of around $13.4 \mathrm{~nm}[1]$.

Several aspects of EUVL still need to be improved to meet the requirements for highvolume manufacturing. In particular, defects that introduce errors in the printed image are one of the major yield limiters for mask blanks. Several defect reduction schemes including the development of a cleaner deposition process [2] or optimization of the reflective multilayer deposition process to allow smoothing of particles or pits [3] have been suggested, but it is questionable if these techniques alone will be sufficient to meet the very stringent requirements for EUVL reticle production [4]. In order to alleviate this problem, it has been suggested that mask blank yield can be improved by repairing defective mask blanks [5]. 
It is unique to EUVL that particles even smaller than $30 \mathrm{~nm}$ in diameter that are located on or near the substrate can nucleate defects in the multilayer coating that result in errors in the printed image [6]. These kinds of defects are called phase defects. The local distortion of the multilayer structure associated with phase defects leads to a phase shift of the reflected EUV radiation over the defect, whereas the amplitude of the reflected light is essentially unchanged. It has been suggested to repair these phase defects by heating the multilayer locally with a high-resolution electron beam to activate silicide formation that lead to deformations that cancel out the distortion of the multilayer [5].

The distortions of the multilayer associated with phase defects depend on the shape and size of the particle, and on the details of the multilayer deposition process. Figure 1 shows calculations of the width and height of a surface bump in a multilayer as a function of particle size. In this case the mask blank consists of 50 bilayers with a spherical particle between the multilayer and with the substrate. The calculations were performed assuming an ion-assisted smoothing process described in [3]. The height of the surface bumps is on the order of a few nanometers, and the diameter varies between approximately 75 and $100 \mathrm{~nm}$, depending on the size of the defect. These dimensions lead to the definition of the specific requirements for the interaction of the electron beam with the multilayer for phase defect repair in that the diameter of the depressions has to be less than $100 \mathrm{~nm}$. Mirkarimi et al. [5] demonstrated that an electron beam can heat a multilayer to sufficiently high temperatures so that local multilayer contraction can be observed. However, the electron beam that was used in that initial set of experiments 
was $400 \mu \mathrm{m}$ in diameter, and, as expected, the depression diameters were of similar magnitude.

The objective of this work is to reduce the diameter of the electron-beam-induced depressions which is necessary to develop a viable phase defect repair technique for EUVL mask blanks. In the course of our experimental work, we have found that the diameter of these depressions is 10 to 100 times larger than the nm-sized electron beam diameter. We further determined that the electron beam current that is required to induce surface depressions in standard EUVL mask blanks is lower than previous modeling has suggested $[5,7]$. We have found that both the strains due to the interaction of the electron beam with the multilayer and the substrate, as well as the mechanical response of the mask blank to the electron-beam-induced free strains have to be taken into account to explain these results. In this paper we (i) discuss the physical models describing the interaction of an electron beam with an EUVL mask blank, (ii) present our experimental observations using electron beams of deep sub-micron diameter and compare them to the models, and (iii) refine the list of requirements for the phase defect repair technique.

\section{Procedure}

We will first outline the models and simulation tools that we used to describe the electron beam interaction with a mask blank. We then discuss the experimental setup for testing and validating the models. 


\section{A. Modeling}

We modeled the deformations of an EUVL mask blank consisting of a Mo/Si multilayer reflective coating deposited on a silica substrate upon electron beam exposure. We give an overview of the model in this section and describe the different parts of it in more detail below. The multilayer coating was composed of $\mathrm{N}$ bilayers with a bilayer thickness of $\Lambda=7.0 \mathrm{~nm}$. The total film thickness is then the product of $\mathrm{N}$ and $\Lambda$.

The transport of the electron beam in the multilayer coating and the corresponding energy deposition were calculated using a Monte Carlo approach [8]. The result corresponds to the energy deposited per unit volume for a single incident electron. This was subsequently convolved with the profile of the incident beam that was assumed to be Gaussian-shaped, to obtain the actual energy deposition profile. The deposited energy can lead to volumetric changes in (i) the multilayer due to molybdenum silicide formation [9] and (ii) in the substrate due to silica compaction [10]. We calculated the stress-free strains associated with these volumetric changes, and then calculated the mechanical deformation of the mask blank using finite element modeling (FEM) to solve for the displacement equations. The volume change in the multilayer was determined by first calculating the temperature profile in the mask blank produced by the electron beam exposure using FEM to solve for the thermal diffusion, and then calculate the molybdenum silicide formation at elevated temperature. The volume change in the 
substrate was determined by calculating the energy dose in the substrate and using literature data to relate the dose to the associated volume compaction [11].

\section{i. Energy deposition due to electron beam}

The electron transport in the multilayer and the corresponding energy deposition were calculated using a Monte Carlo approach [8]. We used the Rutherford model to describe the elastic scattering $[12,13]$ and a modified Bethe equation to describe the inelastic scattering leading to energy loss $[14,15,16]$. We used a single scattering model that handles each scattering event that changes the direction of the electron trajectory separately. The effect of secondary electron production on the energy deposition profiles was found to be negligible.

Calculations were performed for electron beam voltages ranging from 4 to $20 \mathrm{kV}$. The multilayer was modeled as a homogeneous film on a silica substrate. The materials parameters for the film corresponded to the appropriately weighted averages of Mo and $\mathrm{Si}$, assuming that the ratio of the Mo layer thickness to the bilayer thickness was $\Gamma=0.4$. These values are listed in Table I. The stoichiometry of the equivalent single film is $\mathrm{Mo}_{0.46} \mathrm{Si}_{0.54}$

\section{ii. Temperature profile and silicide formation}


The temperature profile produced by the electron beam exposure was calculated using the commercial FEM software FlexPDE [17]. We considered the case of exposing the mask to a stationary electron beam. For exposure times of $1 \mathrm{~s}$ or larger, the response time of the system of the order of microseconds is much smaller than the duration of the stationary beam, so that we modeled the thermal transport using the time-independent diffusion equation. In cylindrical coordinates [18],

$\frac{1}{\mathrm{r}} \frac{\partial}{\partial \mathrm{r}}\left(\kappa \frac{\partial \mathrm{T}}{\partial \mathrm{r}}\right)+\frac{\partial}{\partial \mathrm{z}}\left(\kappa \frac{\partial \mathrm{T}}{\partial \mathrm{z}}\right)+\mathrm{H}(\mathrm{r}, \mathrm{z})=0$.

where $\kappa$ is the thermal conductivity, and $\mathrm{H}$ is the rate of energy deposition by the electron beam. $\mathrm{r}$ is the radial coordinate in the plane of the multilayer, and $\mathrm{z}$ is the vertical coordinate perpendicular to the substrate. For the heat source we imported the tabulated results from the Monte Carlo simulations into the FEM calculation, and interpolated this data onto the finite element mesh.

Boundary conditions were imposed in which the top surface of the mask was thermally insulated, and the sides and bottom surface were fixed at room temperature $\left(300^{\circ} \mathrm{K}\right)$. The thickness of the substrate was chosen to be $100 \mu \mathrm{m}$, and the size of the simulation domain in the radial dimension was $100 \mu \mathrm{m}$. The dimensions were sufficiently large to eliminate any effects due to the boundary conditions on the sides and bottom surface of the mask. 
As above, we treated the multilayer coating as a homogeneous film having material properties that were a weighted average of $\mathrm{Mo}$ and $\mathrm{Si}$. The thermal conductivity of the substrate was assumed to be isotropic. However, the thermal conductivity of the multilayer is generally different in- and out-of-plane and was estimated from literature date, as described in Appendix I. As expected, our calculations show that the thermal conductivity of the multilayer is much smaller than the thermal conductivity of the corresponding bulk materials due to strong interface scattering: The mean free path of an electron in Mo is $23 \mathrm{~nm}$ [19], whereas the nominal Mo thickness in the multilayer is less than $3 \mathrm{~nm}$. The thermal properties we used are listed in Table II.

Finally, we calculated the free-strains associated with the silicide formation at the multilayer interfaces. The reaction of Mo and Si is rate-limited by thermally-activated interdiffusion. The width of the interlayer, $\mathrm{w}$, increases with time according to

$\mathrm{w}^{2}=\mathrm{w}_{0}^{2}+2 \mathrm{Dt}$

where $\mathrm{w}_{0}$ is the as-deposited interlayer width, and $\mathrm{t}$ is the time. $\mathrm{D}$ is the diffusivity given by

$\mathrm{D}(\mathrm{T})=\mathrm{D}_{0} \exp \left(-\mathrm{E}_{\mathrm{a}} / \mathrm{kT}\right)$ 
where $\mathrm{T}$ is the temperature, $\mathrm{E}_{\mathrm{a}}$ is the activation energy, $\mathrm{k}$ is the Boltzmann constant, and $\mathrm{D}_{0}$ is a constant. It has been observed that interfaces where Mo is deposited on Si behave differently from the interfaces where $\mathrm{Si}$ is deposited on Mo [9]. For interfaces with Mo on top of $\mathrm{Si}, \mathrm{w}_{0} \approx 0.5 \mathrm{~nm}$ and $\mathrm{D}_{0} \approx 5 \mathrm{~cm}^{2} / \mathrm{s}$, and for interfaces with $\mathrm{Si}$ on on top of Mo, $\mathrm{w}_{0} \approx 1.0 \mathrm{~nm}$ and $\mathrm{D}_{0} \approx 50 \mathrm{~cm}^{2} / \mathrm{s}$ [9]. The formation of the silicide interlayer produces a contraction of the multilayer period given by

$\Delta \Lambda=\Lambda_{0}-\alpha\left(\mathrm{w}-\mathrm{w}_{0}\right)$.

Here, $\alpha$ is the contraction factor that depends on the particular silicide compound that is formed. In this study we have used $\alpha=0.38$, which corresponds to the contraction that occurs upon the formation of $\mathrm{MoSi}_{2}$.

\section{iii. Substrate compaction}

The fractional volume change or dilatation that results when fused silica is irradiated with high-energy particles has been investigated for many years. The dependence of the compaction on the fluence has been studied for several kinds of irradiations, including neutron, light-ion, electron, and $\gamma$-rays [10]. The detailed physical origin of the compaction is not well understood [20], but the effect has been recognized as a potential issue in a variety of areas, such as optical elements for photolithography or oxide films 
used in the semiconductor industry. It has also been suggested that the change in refractive index that accompanies the dilatation could be used to fabricate waveguides [21].

We used the results of the energy deposition from the Monte Carlo simulations, the electron current, and the pulse length to determine the dose as a function of position in the glass substrate. We then use the experimental data reported by Norris et al. that describes the dilatation effects in fused silica due to 2 to $18 \mathrm{keV}$ electron irradiation [11] in order to determine the stress-free strains in the substrate due to electron exposure. We assume for the stress-free strains due to silicide formation discussed in the previous Section to occur only in the vertical z-direction, whereas the volume change associated with substrate compaction is isotropic.

We do not expect that pure thermal heating of the mask blank by the electron beam will lead to substrate compaction, but rather that energetic particles are necessary to induce dilatations. This agrees with observations by von Bibra et al. that silica compaction actually relaxes upon longer thermal annealing [22]. The annealing times needed to relax the structure are much longer than our electron beam exposure times.

\section{iv. Mechanical deformation of the mask blank}


Both silicide formation and substrate compaction lead to volumetric changes that can formally be characterized by stress-free strains. Stress-free strains describe the deformations of the material if it were free to change its volume. However, the mask blank material cannot contract or expand freely, leading to extrinsic stresses. By considering the mechanical response of the material, we can calculate the constrained strains in the mask blank, which describe the actual mechanical deformation due to electron beam exposure.

For the mechanical calculations we assumed that both the multilayer and the substrate are isotropic materials that behave perfectly elastic. In cylindrical coordinates, the differential equations of equilibrium arising from a force balance in the material are [23]

$$
\begin{aligned}
& \frac{\frac{\partial}{\partial \mathrm{r}}\left(\mathrm{r} \sigma_{\mathrm{r}}\right)}{\mathrm{r}}-\frac{\sigma_{\mathrm{t}}}{\mathrm{r}}+\frac{\partial \tau_{\mathrm{rz}}}{\partial \mathrm{z}}=0, \text { and } \\
& \frac{\frac{\partial}{\partial \mathrm{r}}\left(\mathrm{r} \tau_{\mathrm{rz}}\right)}{\mathrm{r}}+\frac{\partial \sigma_{\mathrm{z}}}{\partial \mathrm{z}}=0 .
\end{aligned}
$$

$\sigma_{\mathrm{z}}, \sigma_{\mathrm{r}}$, and $\sigma_{\mathrm{t}}$ are the stresses in the $\mathrm{z}-, \mathrm{r}-$, and theta-directions, respectively, and $\tau_{\mathrm{rz}}$ is the shear stress. The constrained strains can then be calculated using the constitutive relations of the materials based on Hooke's law. The mechanical materials properties we 
assumed in the calculations are listed in Table III, and we assume that they do not change during the phase transformation of the material.

\section{B. Experimental procedure}

We deposited Mo/Si multilayer films on $6.3 \mathrm{~mm}$-thick and $25.4 \mathrm{~mm}$-wide ultra-lowexpansion (ULE) substrates [24] using an ion beam sputtering process process [3]. For most of the experiments we deposited 50 bilayers, and for some experiments we deposited 25 and 99 bilayers instead. In all cases we deposited an extra silicon layer, so that all multilayers had silicon layers on the top and at the bottom. The bilayer thickness was $\sim 7.0 \mathrm{~nm}$, and the ratio of the Mo layer thickness to the bilayer thickness, $\Gamma$, was approximately 0.4 .

The Mo/Si samples were irradiated with a mono-energetic electron beam with an energy between 4 and $15 \mathrm{keV}$. For the different exposures, the electron beam current was varied between 1 and $1000 \mathrm{nA}$. The electron beam was stationary, and the exposure time was 1 s. We used two different tools to generate the electron beam. In the first set of experiments, we focused the electron analysis beam of a JEOL 8200 electron microprobe onto the sample surface. This electron beam tool is capable of achieving electron currents of $1 \mu \mathrm{A}$, and the beam diameter is $400 \mathrm{~nm}$ or larger. We estimated the electron beam diameter by imaging gold islands on a carbon substrate, and taking the beam diameter as the minimum distance of two Au edges that can be resolved. In the second 
set of experiments, we focused the electron beam of a JEOL JSM-7000F field emission scanning electron microscope (SEM) onto the sample surface. With this electron beam tool we achieved currents up to $150 \mathrm{nA}$ at $10 \mathrm{keV}$, and the diameter of the beam was smaller than $20 \mathrm{~nm}$. The dependence of the electron beam diameters of these tools on electron energy and current are described in more detail in the next Section.

The surface profiles were measured using a ZYGO white light interferometer [25] and, for comparison and calibration, using an atomic force microscope (AFM). The peak depression was taken as the depth of a surface depression, and the full-width-at-halfmaximum (FWHM) as the width.

We studied the exposure of multilayers of different thicknesses to electron beams of various parameters as described in more detail in the following Section. In a subset of the experiments, we pre-annealed the multilayer prior to electron beam exposure for 2 hours at $150^{\circ} \mathrm{C}$ in a vacuum of $10^{-8}$ Torr. To test the effect of the substrate on the mask blank deformation, we replaced the ULE substrate with silica, Zerodur [26], or crystalline silicon substrates, and we also exposed ULE substrate coated just with a $4.5 \mathrm{~nm}$ bilayer of Mo and Si without a multilayer in some experiments. In a final subset of experiments, we replaced the standard $\mathrm{Mo} / \mathrm{Si}$ multilayer film with a multilayer with a thin $\mathrm{B}_{4} \mathrm{C}$ interlayer between each interface. 


\section{Experimental Results}

Figure 2 shows estimates of the electron beam diameter of the microprobe and the SEM. We were able to achieve much smaller electron beam diameters in the SEM, but on this tool the currents were limited to $70 \mathrm{nA}$ at $4 \mathrm{keV}, 150 \mathrm{nA}$ at $10 \mathrm{keV}$, and $200 \mathrm{nA}$ at 15 $\mathrm{keV}$. The microprobe allowed using currents up to $1 \mu \mathrm{A}$, but the diameter was larger than $400 \mathrm{~nm}$.

Figure 3 (a) shows the depression profile of a Mo/Si multilayer that was irradiated for one second with a $12 \mathrm{keV}$ electron beam from the microbeam tool using a current of 20 nA, obtained using a ZYGO white light interferometer. Since the depression diameter of 2 to $2.5 \mu \mathrm{m}$ is comparable to the lateral resolution of the interferometer of approximately $1 \mu \mathrm{m}$, we compared these results with surface profiles measured using an atomic force microscope (AFM). The AFM has better lateral resolution, but the measurements are more time-intensive since the small field of view of an AFM makes cross registration to locate the depressions more difficult, and the scan time is substantially larger. We compared the depths and widths of the depressions obtained with the interferometer with the measurements from the AFM and found that the results agree within $20 \%$ for depressions larger than $1.5 \mu \mathrm{m}$. In the following, all depths and widths measurements for depressions larger than $1.5 \mu \mathrm{m}$ were measured using the interferometer, and smaller depressions were measured using AFM. 
Figure 4 shows the depth of surface depressions of an ion-beam-deposited $\mathrm{Mo} / \mathrm{Si}$ multilayer with 50 bilayers on a ULE substrate that was irradiated for one second with either a 10 or $12 \mathrm{keV}$ electron beam using the microprobe, as a function of the electron beam current. The widths of the surface depressions were approximately $2.5 \mu \mathrm{m}$. We found that both higher electron energies and larger electron beam currents lead to deeper surface depressions. For very high currents the depression depth tends to saturate. We performed similar experiments on magnetron-deposited Mo/Si multilayers and found that the results were similar to the ion-beam-deposited multilayers.

Figure 5 shows the depth of surface depressions of a Mo/Si multilayer film with 50 bilayers on a ULE substrate that was irradiated with an electron beam for one second using the SEM, as a function of electron beam current. The depressions were produced using $10 \mathrm{keV}$ and $15 \mathrm{keV}$ electron beams with diameters in the range from $5 \mathrm{~nm}$ to 15 $\mathrm{nm}$, as shown in Figure 2 (b). No depressions were observed with a $4 \mathrm{keV}$ electron beam for currents up to $70 \mathrm{nA}$. We again found that both higher electron energies and larger beam currents lead to deeper surface depressions, and that the depression depth tends to saturate for high currents. Figure 6 shows the corresponding FWHM of the depressions. We observe that the depression diameters far exceed the electron beam diameters.

It can be expected that the relative contribution of the multilayer contraction and the substrate compaction to the stress-free strains will change depending on the multilayer thickness, since for a thicker multilayer, the electron beam needs to penetrate a thicker film before it reaches the substrate. Figure 7 shows the depths of surface depressions in 
$\mathrm{Mo} / \mathrm{Si}$ multilayers films with 25, 50, and 99 bilayers on ULE substrates that were irradiated with a $10 \mathrm{keV}$ electron beam for one second using the microprobe. We found that the depression depth generally decreases with increasing multilayer thickness. When we exposed a blank ULE substrate coated with just one Mo/Si bilayer to prevent surface charging effects to an electron beam, we also observed surface depressions. Figure 8 shows the depression depth as a function of the electron energy for an electron beam current of $10 \mathrm{nA}$ and exposure times of 1 second. The electron energy was varied between 2 and $10 \mathrm{keV}$, and the depression depth was found to increase with increasing electron energy. Since no multilayer is present, these depressions are solely due to substrate compaction.

Another way to vary the relative contribution of the multilayer contraction and the substrate compaction is to slow the silicide formation process by incorporating an interlayer between each silicon and molybdenum interface. We used $2 \mathrm{~nm}$ of $\mathrm{B}_{4} \mathrm{C}$ as an interlayer by depositing $50 \mathrm{Si} / \mathrm{B}_{4} \mathrm{C} / \mathrm{Mo} / \mathrm{B}_{4} \mathrm{C}$ stacks, of which the bottom and top layers were silicon. This multilayer was exposed to a $10 \mathrm{keV}$ electron beam for 1 second. Figure 9 shows the depression depth as a function of the electron beam current, and it can be seen that the electron current requirements for interface-engineered multilayers with $\mathrm{B}_{4} \mathrm{C}$ diffusion barriers is more stringent. Note that this result is more important for gaining a physical understanding of the contraction mechanism than for the technological development of a phase defect repair process since while a $\mathrm{B}_{4} \mathrm{C}$ interlay is being considered seriously for multilayer-coated optics, it is not as important for multilayercoated mask blanks. 
The substrate material is expected to affect the shape and depth of the surface depressions since it both cools the irradiated multilayer, thereby reducing the amount of silicide formation, and it possibly exhibits volumetric compaction. We irradiated $\mathrm{Mo} / \mathrm{Si}$ multilayer films with 50 bilayers on fused silica, ultra-low-expansion glass (ULE), Zerodur, and crystalline Si substrates with a $10 \mathrm{keV}$ electron beam for 1 second. ULE is fused silica doped with Ti to reduce the coefficient of thermal expansion [24], and Zerodur is a multi-phase zero-expansion glass ceramic [26]. We did not find any differences in the surface depressions of irradiated multilayers on fused silica and ULE substrates. However, for samples with Zerodur substrates, significantly larger electron currents were required to induce surface depressions than for samples with ULE substrates, and eventually the surface started to buckle. Note that this is not expected to be an issue since ULE is currently the leading candidate material for the mask substrate. For $\mathrm{Si}$, we did not observe any surface changes for currents up to $1 \mu \mathrm{A}$, which is the highest current we used in our experiments.

Finally, to further determine if substrate compaction or multilayer contraction dominates, we modified the initial silicide thickness by annealing a Mo/Si multilayer film with 50 bilayers on a ULE substrate for 2 hours at $150^{\circ} \mathrm{C}$. We observed that the annealing step reduced the wavelength at which the reflectance peaks by $0.1 \mathrm{~nm}$, which corresponds to a $2.5 \mathrm{~nm}$ contraction of the whole multilayer film. We then irradiated this film for 1 second with a $10 \mathrm{keV}$ electron beam with currents up to $100 \mathrm{nA}$. According to Equation (2), this sample would deform differently if multilayer contraction dominated. We 
repeated this experiment with a film that was not annealed, and we did not observe any differences in the surface depressions, suggesting that substrate compaction dominates.

\section{Modeling Results}

We performed calculations of the energy deposition profiles for electron energies ranging from 5 to $20 \mathrm{keV}$ for various multilayer thicknesses. We found strong scattering within the multilayer coating and, for high energies, also scattering within the substrate. This causes the deposition profile to expand laterally with depth, producing the well-known tear-drop-shaped contours [27]. The energy deposition profiles and the amount of energy that is transported away by backscattered electrons depend on the electron energy and multilayer thickness. Figure 10 shows the fraction of the incoming electron energy that is deposited into the substrate, deposited into the multilayer, and backscattered, as a function of the multilayer thicknesses for different electron energies. The amount of backscattered energy varies between 15 and $20 \%$. As expected, the fraction of energy that is deposited into the substrate increases with increasing electron energy.

The energy deposition by the electron can lead to deformation of the mask blank due to both heating of the multilayer that leads to multilayer contraction induced by silicide formation, and compaction of the substrate. Figure 11 shows the calculated peak temperature rise as a function of electron current for a multilayer consisting of 50 bilayers, irradiated by 4,10 , and $15 \mathrm{keV}$ electrons. The peak temperature is an 
approximate measure for the onset of stress-free deformations in the multilayer. For the electron beam diameters in these simulations, we used the measured diameters of the SEM, see Figure 2 (b).

It is convenient to describe the mask blank deformation by stress-free strains, and then calculate the mechanical response of the system separately. To demonstrate the effect of the mechanical response, we calculated the shape of the surface depression as a function of the distance of a contracting volume element from the surface. Figure 12 shows the peak depression depth and FWHM as a function of the distance of the top surface of this volume element from the mask blank surface. The volume element was assumed to have a cylindrical shape of diameter $50 \mathrm{~nm}$ and height $50 \mathrm{~nm}$, located at different positions along the $\mathrm{z}$ axis in the multilayer, and is contracting uniformly in the $\mathrm{z}$-direction by $1 \%$. The mechanical response of the surrounding material leads to significant differences between the free strains and the constrained strains. Figure 13 shows the constrained strains as a function of the $\mathrm{z}$ position along the center of the cylinder for the case that a volume element in the shape of a cylinder of radius $50 \mathrm{~nm}$ that spans the thickness of the multilayer contracts in the $\mathrm{z}$ direction by $1 \%$. In both these calculations, the multilayer thickness was assumed to be $350 \mathrm{~nm}$, corresponding to $50 \mathrm{Mo} / \mathrm{Si}$ bilayer pairs.

Using our full set of simulation tools, we found that our calculations agree with the experimental observation well. In Figures 4 and 8 we have overlaid simulation results over the measurements for the case of the wide electron beam, and in Figures 5 and 6 for the narrow electron beam. In the simulations we used the same electron beam 
parameters, such as energy, current, and diameter, and mask blank configurations as in the experiments. We were further able to predict the outcome for the case of exposing a multilayer of different thicknesses to the wider electron beam, see Figure 7.

\section{Discussion}

Through extrapolation of experimental annealing data [9], we estimated that in order to achieve appreciable silicide formation in the multilayer within one second, a temperature rise of at least $420^{\circ} \mathrm{C}$ over room temperature is required. According to Figure 11, these temperatures are achieved only for currents larger than $160-200 \mathrm{nA}$. For higher electron energies, generally larger currents are required to achieve this temperature because a larger fraction of the energy is deposited into or near the substrate.

We have discovered that different physical mechanisms dominate during the electron beam exposure of Mo/Si multilayers, primarily depending on the electron voltage and current, and multilayer thickness. At lower currents, which are currents much less than $100 \mathrm{nA}$, our calculations show that the temperature rise in the multilayer is too small to induce significant multilayer contraction by silicide formation. However, if the multilayer is thin enough and the electron energy is large enough, a fraction of the electron energy is deposited into the substrate, leading to its contraction. For example, we calculated that only approximately $5 \%$ of the energy of a $10 \mathrm{keV}$ electron beam is deposited into the substrate of a mask blank with a $350 \mathrm{~nm}$-thick multilayer film, but this 
energy is enough to induce significant multilayer contractions. However, virtually no electron energy is deposited into the substrate for a $4 \mathrm{keV}$ electron beam up to $70 \mathrm{nA}$ for a multilayer of the same thickness, so that no substrate compaction is expected. This agrees with our experimental findings shown in Figure 7. We further noticed that for a $10 \mathrm{keV}$ electron beam, substrate compaction is significant for 25 and 50 bilayer films, but not for 99 bilayer films.

At higher currents, which are currents much larger than $100 \mathrm{nA}$, our calculations show that the temperature of the multilayer rises significantly, see Figure 11, and that multilayer contraction is expected. For the case of the thinner multilayers consisting of 25 and $50 \mathrm{Mo} / \mathrm{Si}$ bilayers, the multilayer contraction is concurrent with the substrate compaction for an electron beam at $10 \mathrm{keV}$. For the thicker multilayer made up of 99 Mo/Si bilayers, only the multilayer contraction is observed.

Our models also agree with the dependence of the surface depressions on the substrate materials. For example, crystalline silicon substrates are not expected to compact under electron beam irradiation. Further, Si is a good heat sink, preventing the heating of the multilayer, so that no deformation of the multilayer on $\mathrm{Si}$ is expected during electron beam exposure up to $1 \mu \mathrm{A}$, as was observed in the experiments. For a Zerodur substrate that has thermal properties more similar to silica than $\mathrm{Si}$, a small amount of compaction has been observed [28]. 
Our results are also consistent with results reported by Mirkarimi et al. [5]. In their experiments, an electron beam was used that was $400 \mu \mathrm{m}$ in diameter. They reported a wavelength shift in the reflectance spectrum of the multilayer, and showed that their experimental findings can be explained by multilayer contraction, whereas in our experiments using a much smaller electron beam and a much higher current density, substrate compaction usually dominated. Our calculations show that this different behavior is due to the different electron beam diameters. For a larger electron beam, a significantly lower current density is required to heat the multilayer to a certain temperature than for a narrowly-focused electron beam, because in the case of a large diameter, the multilayer is cooled primarily through vertical heat conduction into the substrate, whereas in the case of a small diameter, the multilayer is cooled much more efficiently by both heat conduction vertically into the substrate as well as laterally into the surrounding multilayer. The energy dose deposited into the substrate is smaller for large-diameter electron beams, and substrate compaction is negligible. This different behavior could be demonstrated directly if the absence of the shift in the reflectivity spectrum could be measured, but tools with the required micrometer spatial resolution are not available to us yet.

Our conventional annealing experiments show that multilayer contraction associated with silicide formation can be observed even at temperatures as low as $150^{\circ} \mathrm{C}$, provided that the annealing time is increased to 2 hours or more. We observed similar depressions for pre-annealed and un-annealed multilayers of $50 \mathrm{Mo} / \mathrm{Si}$ bilayers that were irradiated with electron currents up to $100 \mathrm{nA}$. This again indicates that the substrate is responsible to 
the electron-beam-induced depressions, since a pre-anneal would change the initial silicide thickness, $\mathrm{w}_{0}$, so that according to Equation (2) the film would contract differently if multilayer contraction would dominate.

When interface-engineered multilayers with $\mathrm{B}_{4} \mathrm{C}$ diffusion barriers are irradiated by an electron beam, a larger electron current is required to achieve the same depression depths than without diffusion barriers, as shown in Figure 9. Since we expect substrate compaction to be the dominant deformation mechanism in this case, and it is expected that $\mathrm{B}_{4} \mathrm{C}$ diffusion barriers primarily retard only the molybdenum silicide formation, this results is surprising at first glance. This behavior can be better understood if the different mechanical properties of interface-engineered multilayers [29] are considered. $\mathrm{B}_{4} \mathrm{C}$ is less compliant than Mo and amorphous silicon [30], so it is expected that $\mathrm{B}_{4} \mathrm{C}$-interfaced engineered multilayers have a larger Young's modulus than multilayers without interlayers, and our simulations show that this leads to shallower surface depressions, which we observed in the experiments.

Besides pointing out the importance of substrate compaction, our work also demonstrates the significance of the mechanical response of the whole mask blank. Mechanical effects come into play for smaller affected zones, and are less important for larger affected zones, as in reference [5]. We found that the further a contracting volume element is located away from the multilayer surface, the shallower and wider the surface depressions become. This agrees with the theory of contracting inclusions in semiinfinite solids [31], stating that in the presence of an contracting particle, the 
displacement of a free surface is the same as that of the equivalent plane in an infinite solid, increased by a factor $4(1-v) . \quad v$ is the Poisson's ratio of the particle and the surrounding material. In an infinite solid, displacements in the material surrounding a contracting inclusion are smaller for larger distances from the particle, which explains the observation of shallower surface depressions for contractions further away from the surface. Further, shells of constant displacements have a larger radius of curvature for larger distances from the particle, rationalizing the increase in diameter of the surface depressions.

Due to this mechanical phenomenon, substrate compaction generally leads to surface depressions that are larger in diameter than depressions due to multilayer contraction. This along with the fact that the electron beam is significantly widened by the time it reaches the substrate due to scattering in the multilayer explains why we observed surface depressions that were much larger in diameter than the diameter of the electron beam. In order to achieve surface depressions of smaller diameters, it is desirable that the depressions are confined to the deformation of the multilayer and are not due to the compaction of the substrate.

The mechanical response of the mask blank material also has the effect that the surface depressions are primarily due to deformations near the surface, as shown in Figure 13. Even if the free strains are constant through the thickness of the multilayer stack, as assumed in the calculations for Figure 13, most of the substrate deformation is taking place at the top and bottom surfaces of the multilayer. The top surface is free to move, 
and the bottom surface is less constrained since the substrate material is more compliant than the multilayer. This non-uniform displacement throughout the multilayer stack leads to an undesirable shift of the reflectance curve in frequency space, and this shift is potentially larger than previously expected [5]. The extent of this effect is not yet clearly understood and will need to be investigated in more detail, for example through printability modeling.

\section{Summary and Conclusions}

In summary, we have developed a suite of models that describe the interaction of an electron beam with a Mo/Si multilayer on a substrate, and the models are consistent with all of our experimental findings. We have shown that standard EUVL mask blanks consisting of a Mo/Si multilayer on a glass substrate can be deformed by an electron beam at relatively low currents below $20 \mathrm{nA}$. However, the depression diameters are more than 20 times larger than the electron beam diameters, which were less than $20 \mathrm{~nm}$, and this is not sufficient for phase defect repair of EUVL mask blanks. We found that the deformations are primarily due to the compaction of the substrate and not the multilayer, which inevitably leads to large depression diameters.

In order to realize phase defect repair for EUVL mask blanks, substrate compaction due to overspill of the electron beam into the substrate needs to be prevented. Overspill can potentially be prevented by (i) using a lower energy electron beam, (ii) a thicker 
multilayer, (iii) using an underlayer between the multilayer and the substrate that absorbs the beam spillover, or (iv) using a different substrate material. All these solutions have their own advantages and disadvantages. For example, the solution of using lower energies might be technology-limited since it is generally more difficult to focus a highcurrent electron beam at lower energies than at higher energies. On the other hand, using a thicker multilayer or an underlayer is economically more expensive and could also lead to additional defects in the multilayer. Further, an underlayer would have to have a low thermal conductivity that is at least comparable to the substrate to prevent too efficient heat dissipation that would increase the electron beam current requirements, and the underlayer would also have to have sufficiently low surface roughness for EUVL mask blanks.

Further, our simulations show that phase defect repair requires using an electron beam source with higher currents in a small spot diameter than was accessible to us. Both higher electron currents and the prevention of substrate compaction are necessary requirements for phase defect repair for EUVL mask blanks. In case this can be realized, it still needs to be demonstrated that the reflectance variation over the repaired defect is sufficiently small so that the defect can be considered corrected. Our mechanical simulations have identified a new challenge that even though the stress-free strains are uniform through the multilayer thickness, the constrained strains will not be uniform, which could lead to a significant drop in the amplitude of the reflected light since the multilayer locally does not match the wavelength of the incoming radiation anymore. 
Finally, our work demonstrates that there are still significant technical challenges to making EUVL multilayer phase defect repair a manufacturable technique. We believe that EUVL could still become fit for high-volume manufacturing if (i) the multilayer smoothing process $[3,34]$ continues to advance, and (ii) an EUVL multilayer amplitude defect repair process is developed [35].

\section{Acknowledgements}

We would like to thank Daniel Stearns for helpful discussions, Miles Clift for electron beam exposure work, and Paul Ho and Junjun Liu for useful discussions on the thermal conductivity of multilayer thin films. This work was performed under the auspices of the US Department of Energy by the University of California, Lawrence Livermore National Laboratory under contract No. W-7405-Eng-48. Funding was provided by the Extreme Ultraviolet Limited Liability Company (EUV LLC) under a Cooperative Research and Development Agreement and by Intel Corporation under a Work for Others Agreement. 


\section{References}

[1] E. Spiller, Appl. Phys. Lett. 20, 365 (1972).

[2] J.A. Folta, J. Davidson, C.C. Larson, C.C. Walton, and P.A. Kearney, Proc. SPIE 4688, 173 (2002).

[3] P.B. Mirkarimi, E.A. Spiller, S.L. Baker, V. Sperry, D.G. Stearns, and E.M.

Gullikson, J. Microlith. Microfab. Microsys. 3, 139 (2004).

[4] EUV Mask and Chuck Standard SEMI P38-1102, published by International SEMATECH (2002).

[5] P.B. Mirkarimi, D.G. Stearns, S.L. Baker, J.W. Elmer, D.W. Sweeney, and E.M. Gullikson, J. Appl. Phys. 91, 81 (2002).

[6] E.M. Gullikson, C. Cerjan, D.G. Stearns, P.B. Mirkarimi, and D.W. Sweeney, J.Vac.Sci.Tech. B 20, 81 (2002).

[7] Y.-U. Ko, D.C. Joy, S. Hector, and B. Lu, Proc. SPIE 5038, 293 (2003).

[8] D. Joy, Monte Carlo Modeling for Electron Microscopy and Microanalysis, Oxford University Press, Oxford (1995).

[9] R.S. Rosen, D.G. Stearns, M.A. Viliardos, M.E. Kassner, S.P. Vernon, and Y. Cheng, Appl. Optics 32, 6975 (1993).

[10] W. Primak and R. Kampwirth, J. Appl. Phys. 39, 5651 (1968).

[11] C.B. Norris and E.P. EerNisse, J. Appl. Phys. 45, 3876 (1974).

[12] D.E. Newbury and R.L. Myklebust, Analytical Microscopy 1981, Ed. R.H. Geiss, San Franscisco Press, San Franscisco, p. 91 (1981). 
[13] L. Reimer and E.R. Krefting, Use of Monte Carlo Calculations in Electron Probe Microanalysis and Scanning Electron Microscopy, Ed. K.F.J. Heinrich, D.E. Newbury, and H. Yakowitz (U.S. Dept. of Commerce/National Bureau of Standards), NBS Special Publication \#460, p. 45 (1976).

[14] H.A. Bethe, Phys. Rev. 5, 325 (1930).

[15] M.J. Berger and S.M. Seltzer, Studies in the Penetration of Charged Particles in Matter, Nuclear Science Series Report \#39, NAS-NRC Publication 1133, Natl. Acad.

Sci., Washington, DC, p. 205 (1964).

[16] D.C. Joy and S. Luo, Scanning 11, 176 (1989).

[17] FlexPDE 3.03e1, PDE Solutions, Inc., http://www.pdesolutions.com (2003).

[18] H.S. Carslaw and J.C. Jaeger, Conduction of Heat in Solids, $2^{\text {nd }}$ Edition, Oxford University Press, Oxford (1959).

[19] Ashcroft and Mermin, Solid State Physics, 1976.

[20] N.F. Borrelli, C. Smith, D.C. Allan, and T.P. Seward III, J. Opt. Soc. Am. B 14, 1606 (1997).

[21] K.O. Hill, Y. Fujii, D.C. Johnson, and B.S. Kawasaki, Appl. Phys. Lett. 32, 647 (1978).

[22] M.L. von Bibra, A. Roberts, P. Mulvaney, and S.T. Huntington, J. Appl. Phys. 87, 8429 (2000).

[23] S.P. Timoshenko and J.N. Goodier, Theory of Elasticity, $3^{\text {rd }}$ Edition, McGraw Hill College Division, Columbus, OH (1970).

[24] ULE is an ultra-low-expansion glass fabricated by Corning Inc., Canton, NY. 
[25] We used the ZYGO white light interferometer fabricated by ZYGO Corp., Middlefield, CT.

[26] ZERODUR is a zero-expansion glass ceramic fabricated by Schott Lithotec, Jena, Germany.

[27] T.E. Everhart, R.F. Herzog, M.S. Chang, and W.J DeVore, Proceedings of the $6^{\text {th }}$ International Conference on X-ray Optics and Microanalysis, eds. G. Shinoda, K. Kohra, and T. Ichinojawa, University of Tokyo Press, Tokyo, p.81 (1972).

[28] M. Rajaram and E.J. Friebele, J. Non-Cryst. Sol. 108, 1 (1989)

[29] S. Bajt, High-reflectance interface engineered multilayer, presented at the $6^{\text {th }}$

International Conference on the Physics of X-Ray Multilayer Structures, March 3-7, 2002, Charmoux Mont-Blanc, France.

[30] CRC Handbook of Chemistry and Physics, $84^{\text {th }}$ Edition, CRC Press, Boca Raton, FL (2004).

[31] J.H. Davies, J. Appl. Mech. 70, 655 (2003).

[32] R.J. Anderson, J. Appl. Phys. 67, 6914 (1990).

[33] D.G. Stearns, S.L. Baker, and M.A. Wall, Proc. MRS 403, 183 (1996).

[34] S.P. Hau-Riege, P. B. Mirkarimi, C.C. Walton, V. Sperry, and C. Larson, J. Vac. Sci. Tech. B21, 2466 (2003).

[35] A. Barty, S.P. Hau-Riege, H. Chapman, P. Mirkarimi, D. Sweeney, D. Stearns, M. Clift, and E. Gullikson, to be published in Appl. Opt. 


\section{Appendix: Estimate of the thermal conductivity of Mo/Si multilayers}

Thermal conduction in multilayer thin films with length scales smaller than the electron and phonon mean free paths is generally not very well understood. In the absence of experimental data, we estimate the thermal conductivity in Mo/Si multilayer following the treatment by Anderson [32].

We assume that the multilayer is made up of crystalline Mo and amorphous $\mathrm{Si}(\mathrm{a}-\mathrm{Si}$ ), and that the thermal conductivities of all layers add up linearly. We further assume that the Wiedemann-Franz law holds for Mo thin films, so that there is a linear relationship between the thermal and electrical conductivity in Mo films. We expect that the thermal conductivity in Mo films of a few nm in thickness is substantially lower than in bulk Mo since the electron mean free path in bulk Mo is approximately $23 \mathrm{~nm}$, and interface scattering reduces the mean free path in Mo films substantially. We also assume that the a-Si layers do not contribute significantly to the electrical conductivity of $\mathrm{Mo} / \mathrm{Si}$ multilayers, so that the electrical conductivity is dominated by the Mo films. Finally, we assume that the phonon contribution to the thermal conductivity in Mo scales with electron contribution for varying film thickness since the scale of disorder has the same value for electrons and phonons. Using experimental data for the electrical resistivity as a function of Mo thickness for Mo/Si multilayer [33], we calculate the conductivity of Mo films as a function of thickness, as also shown in Figure 14. As expected, the thermal conductivity of thin-film Mo is significantly lower than for bulk Mo. By neglecting the 
electrical conductivity of the a-Si we overestimated the electrical conductivity of the multilayer and therefore we also overestimated the thermal conductivity of the Mo.

For the a-Si layers we assume that the thickness reduction to a few $\mathrm{nm}$ has little effect on the thermal conductivity since the length scale of disorder is smaller than film thickness, since it is in an amorphous state. We expect the thermal conductivity to be equal or smaller in a thin a-Si film than in bulk a-Si, so that we again overestimated the thermal conductivity of a-Si.

Finally, we assume that simple Fourier heat conduction is valid even on the short length scales in a multilayer. We can then estimate the thermal conductivity of the Mo layer to be $\mathrm{k}_{\mathrm{Mo}}=5.4 \mathrm{~W} / \mathrm{mK}$, and the thermal conductivity the a-Si layer to be $\mathrm{k}_{\mathrm{Si}}=2.5 \mathrm{~W} / \mathrm{mK}$. Then, an upper estimate for the in-plane thermal conductivity at room temperature is

$$
\mathrm{k}_{\mathrm{ML}}=\frac{\mathrm{t}_{\mathrm{Mo}} \mathrm{k}_{\mathrm{Mo}}+\mathrm{t}_{\mathrm{Si}} \mathrm{k}_{\mathrm{Si}}}{\mathrm{t}_{\mathrm{Mo}}+\mathrm{t}_{\mathrm{Si}}}=3.3 \mathrm{~W} / \mathrm{mK} .
$$

If we assume that for out-of-plane heat conduction the interface thermal resistance is negligible, we can calculate an upper estimate for the out-of-plane thermal conductivity of

$$
\mathrm{k}_{\mathrm{ML}}=\frac{\mathrm{t}_{\mathrm{Mo}}+\mathrm{t}_{\mathrm{Si}}}{\left(\frac{\mathrm{t}_{\mathrm{Mo}}}{\mathrm{k}_{\mathrm{Mo}}}+\frac{\mathrm{t}_{\mathrm{Si}}}{\mathrm{k}_{\mathrm{Si}}}\right)}=2.9 \mathrm{~W} / \mathrm{mK} .
$$




\begin{tabular}{|l|c|c|c|}
\hline \multicolumn{1}{|c|}{ Material } & $\rho\left(\mathbf{g} / \mathbf{c m}^{\mathbf{3}}\right)$ & $\mathbf{Z}$ & $\mathbf{W}(\mathbf{g} / \mathbf{m o l})$ \\
\hline Mo/Si coating & 5.47 & 26 & 59.3 \\
\hline ULE substrate & 2.22 & 10 & 60.1 \\
\hline
\end{tabular}

Table I: Values for the mass density $\rho$, the atomic number $\mathrm{Z}$, and the molecular weight W, used in the Monte Carlo simulations of the energy deposition by the electron beam.

\begin{tabular}{|l|c|c|c|}
\hline \multicolumn{1}{|c|}{ Material } & $\begin{array}{c}\kappa_{\text {in-plane }} \\
(\mathbf{W} / \mathbf{m}-\mathbf{K})\end{array}$ & $\begin{array}{c}\kappa_{\text {out-of-plane }} \\
(\mathbf{W} / \mathbf{m}-\mathbf{K})\end{array}$ & $\rho\left(\mathrm{g} / \mathbf{c m}^{3}\right)$ \\
\hline Mo/Si coating & 3.3 & 2.9 & 5.47 \\
\hline Substrate & \multicolumn{2}{|c|}{1.5} & 2.22 \\
\hline
\end{tabular}

Table II: Values for the materials parameters used in the FEM modeling of the multilayer heating. 


\begin{tabular}{|l|c|c|}
\hline \multicolumn{1}{|c|}{ Material } & Poisson's ratio & Young's modulus (GPa) \\
\hline Glass & 0.164 & 72.6 \\
\hline Mo/Si coating & 0.27 & 210.0 \\
\hline
\end{tabular}

Table III: Values for the materials parameters used in the FEM modeling of the mechanical deformation of the mask blank. 
Figure 1: Width and height of the surface bump of a multilayer consisting of 50 bilayers with a spherical particle between the multilayer and the substrate, as a function of particle diameter. The calculations were performed assuming an ion-assisted smoothing process described in [3].

Figure 2: (a) Electron beam diameter of the microprobe at $10 \mathrm{keV}$ as a function of electron beam current. (b) Electron beam diameter of the SEM at 4, 10, and $15 \mathrm{keV}$ as a function of electron beam current. The beam diameters were estimated by determining the resolution of images of gold islands on a carbon substrate.

Figure 3: Depression profile of a Mo/Si multilayer that was irradiated for one second with a $12 \mathrm{keV}$ electron beam from the microbeam tool using a current of $20 \mathrm{nA}$, obtained using (a) a ZYGO while light interferometer and (b) an AFM.

Figure 4: Peak depression in Mo/Si multilayer film on a silica substrate as a function of electron beam current. The depressions were produced by a $10 \mathrm{keV}$ and $12 \mathrm{keV}$ electron beams with diameters in the range from $500 \mathrm{~nm}$ to $3000 \mathrm{~nm}$. Overlaid are peak surface depressions calculated using our models.

Figure 5: Peak depression in Mo/Si multilayer film on a silica substrate as a function of electron beam current. The depressions were produced by $10 \mathrm{keV}$ and $15 \mathrm{keV}$ electron beams with diameters in the range from $5 \mathrm{~nm}$ to $15 \mathrm{~nm}$. No depressions were observed with a $4 \mathrm{keV}$ electron beam for currents up to $70 \mathrm{nA}$. Overlaid are peak surface 
depressions calculated using our models. The calculations predict no depressions with a $4 \mathrm{keV}$ electron beam for currents up to $70 \mathrm{nA}$.

Figure 6: Full-Width-at-Half-Maximum (FWHM) of the depressions discussed in Figure C. The depressions were produced by $10 \mathrm{keV}$ and $15 \mathrm{keV}$ electron beams with diameters in the range from $5 \mathrm{~nm}$ to $15 \mathrm{~nm}$. It is noticeable that the depression diameters far exceed the electron beam diameters. Overlaid are FWHM of surface depressions calculated using our models.

Figure 7: Peak depressions of Mo/Si multilayers films on silica substrates as a function of electron beam current for different multilayer thicknesses. Overlaid are peak surface depressions calculated from the substrate-compaction model.

Figure 8: Peak depression of silica substrate as a function of electron beam voltage. The depressions were produced by a $10 \mathrm{nA}$ electron beam using an exposure time of 5 seconds. Overlaid are peak surface depressions calculated using our models described.

Figure 9: Peak depression in Mo/Si multilayers with and without $\mathrm{B}_{4} \mathrm{C}$ diffusion barriers on a silica substrate as a function of electron beam current. The depressions were produced by a $10 \mathrm{keV}$ electron beam applied for 1 second.

Figure 10: Fraction electron energy that is deposited into substrate, deposited into the multilayer, and backscattered, as a function of multilayer thicknesses for different 
electron energies calculated by Monte Carlo simulations. The Mo/Si bilayer thickness was assumed to be $7 \mathrm{~nm}$.

Figure 11: Calculated peak temperature rise in the mask blank as a function of electron current for a multilayer consisting of 50.5 bilayers, irradiated by 4,10 , and $15 \mathrm{keV}$ electrons. We assumed the SEM beam diameters, see Figure AC (b). Indicated by the horizontal line is the temperature rise of $420^{\circ} \mathrm{C}$, which is the minimum temperature requires to achieve appreciable silicide formation in the multilayer.

Figure 12: Calculated depth and FWHM of the surface depression as a function of distance from the top surface of a contracting volume element. The volume element was assumed to have a cylindrical shape of diameter $50 \mathrm{~nm}$ and height $50 \mathrm{~nm}$, is located at different positions along the $\mathrm{z}$ axis in the multilayer, and is contracting in the $\mathrm{z}$-direction by $1 \%$.

Figure 13: Constrained strains as a function of the $\mathrm{z}$ position along the center of the cylinder for the case that a volume element in the shape of a cylinder of radius $50 \mathrm{~nm}$ that spans the thickness of the multilayer contracts in the $\mathrm{z}$ direction by $1 \%$.

Figure 14: Electrical conductivity of Mo/Si multilayers as a function of Mo thickness [33]. On the right axis, the inferred thermal conductivity of the Mo layers is shown. 


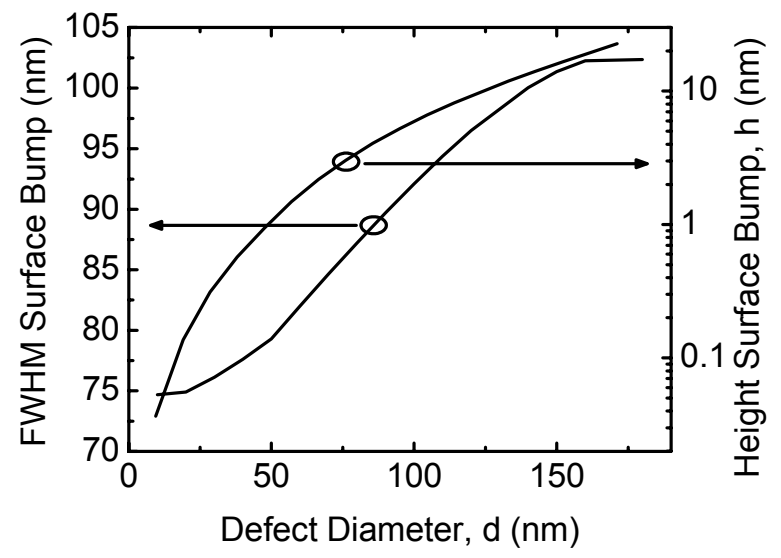

Figure 1 


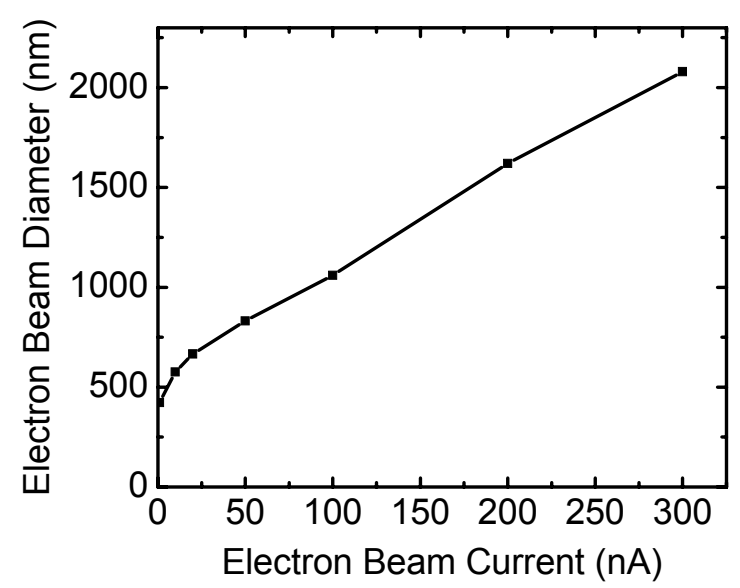

(a)

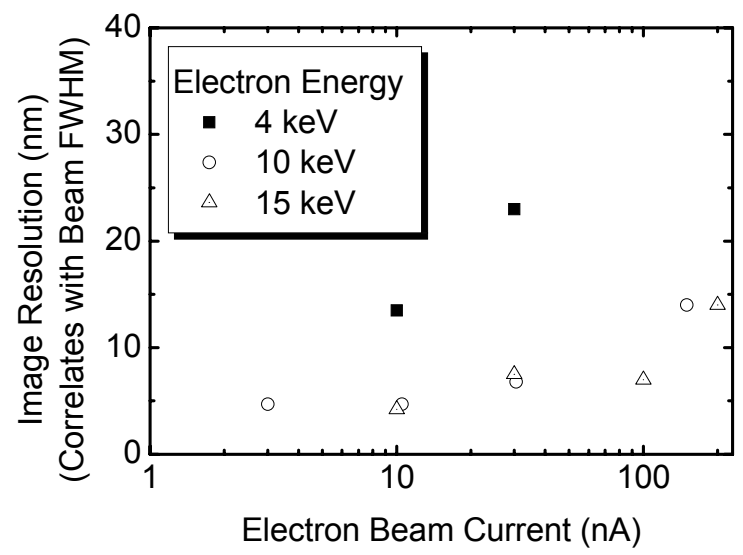

(b)

Figure 2 


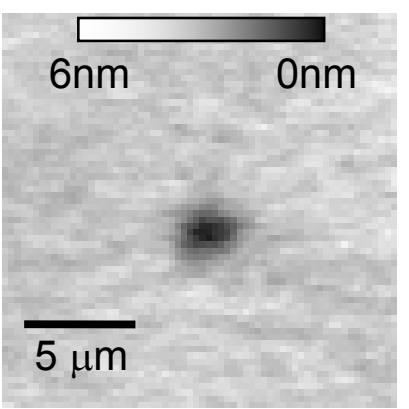

(a)

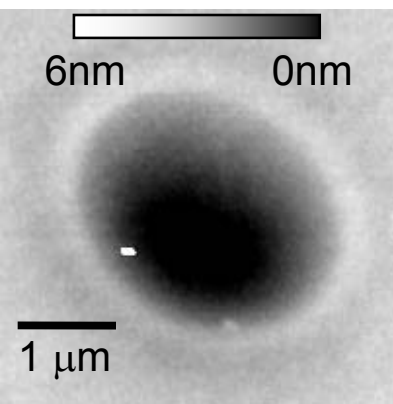

(b)

Figure 3

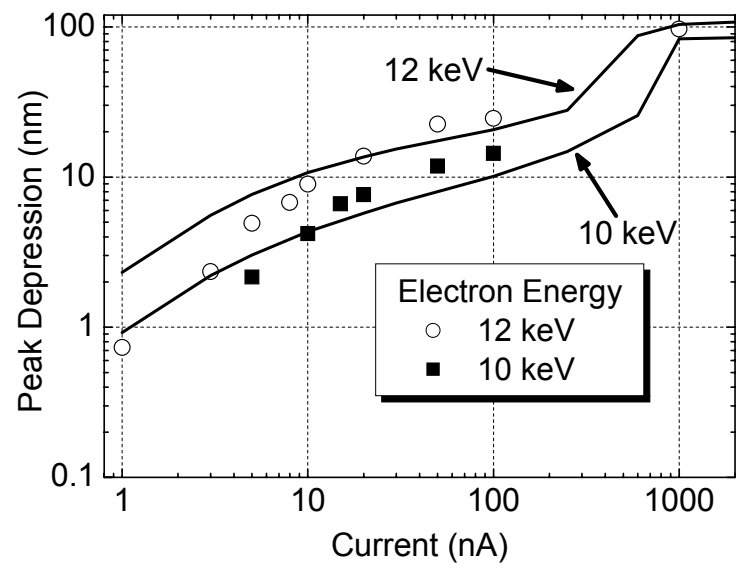

Figure 4 


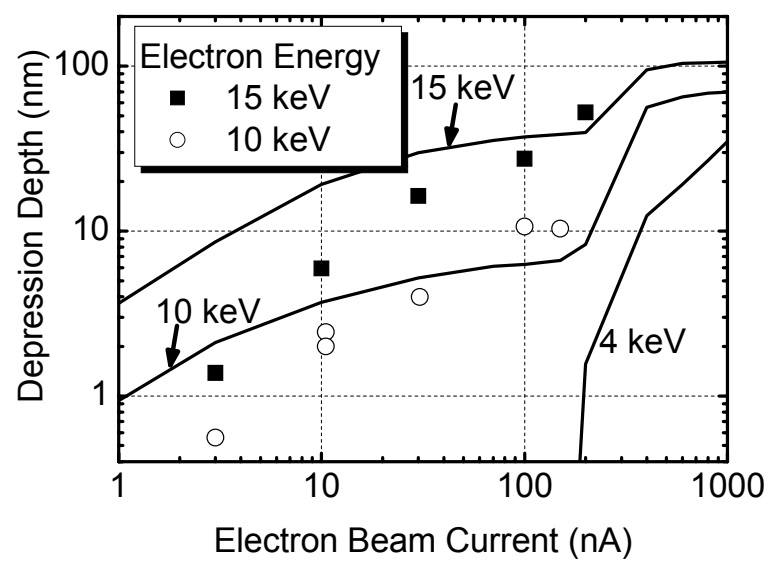

Figure 5

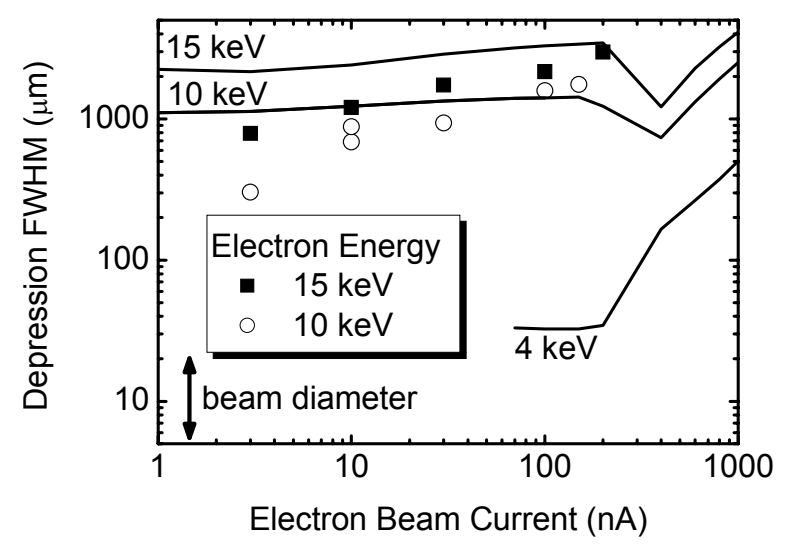

Figure 6 


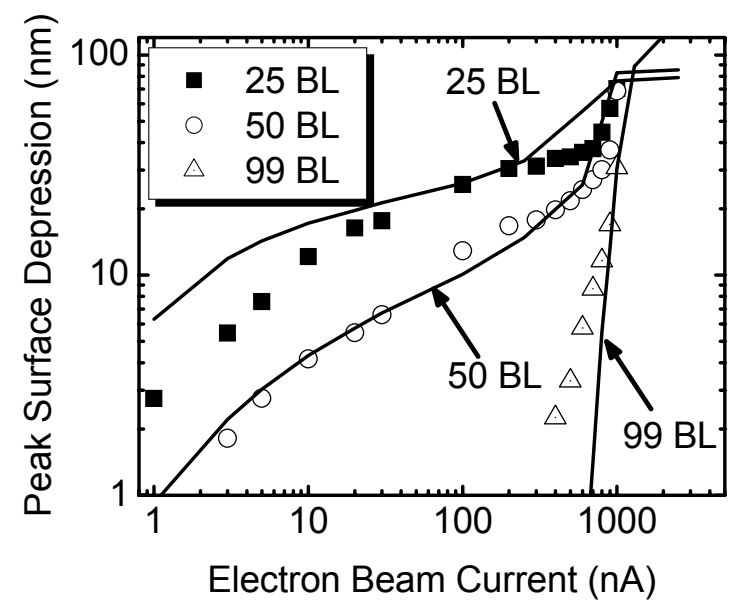

Figure 7

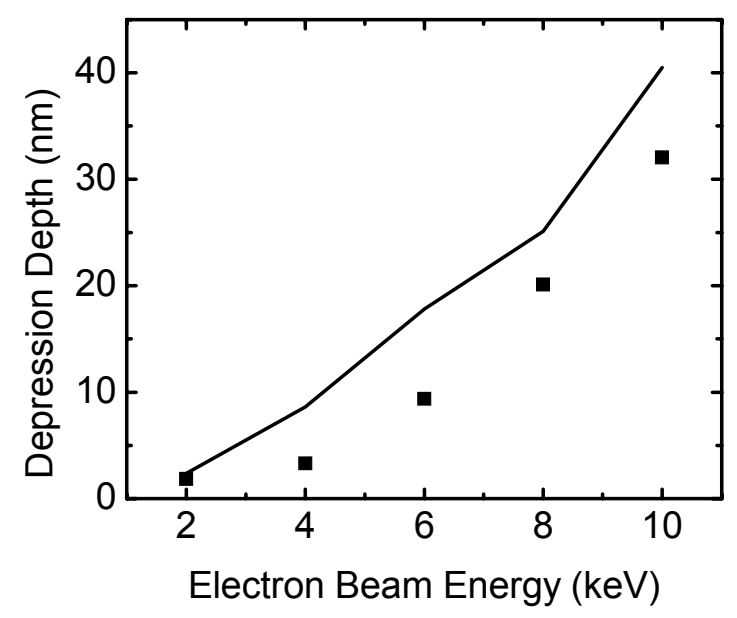

Figure 8 


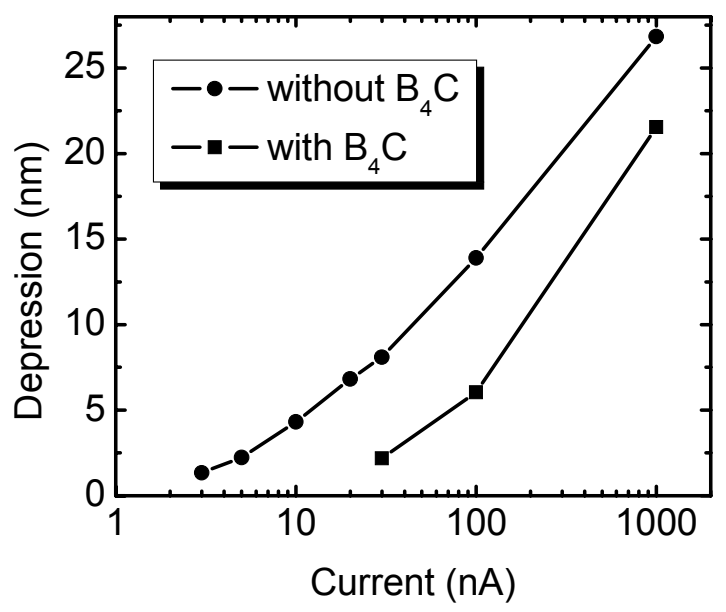

Figure 9

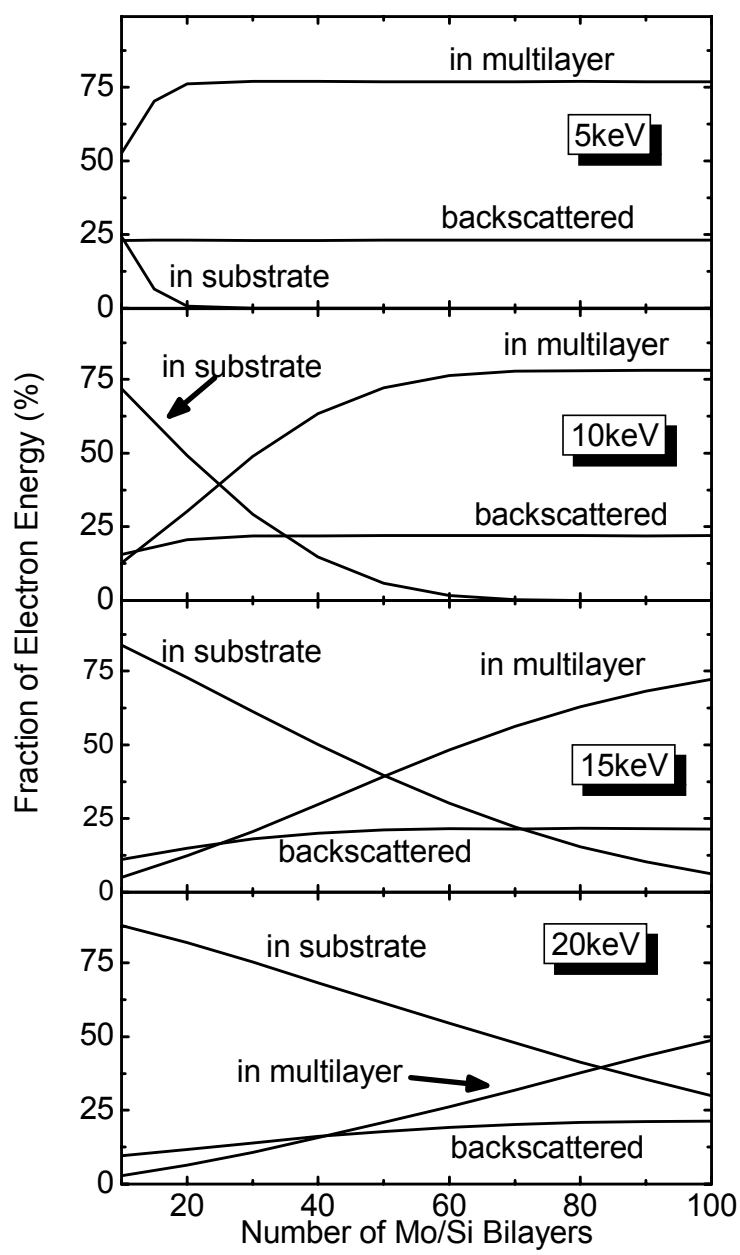


Figure 10

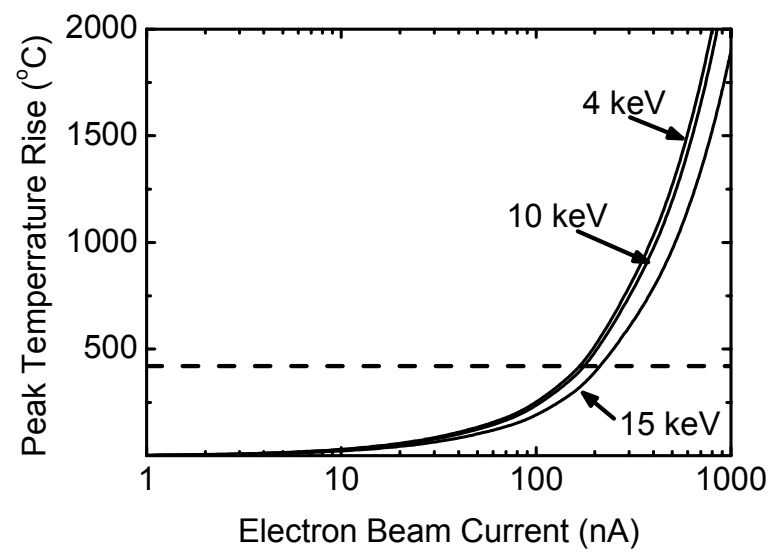

Figure 11

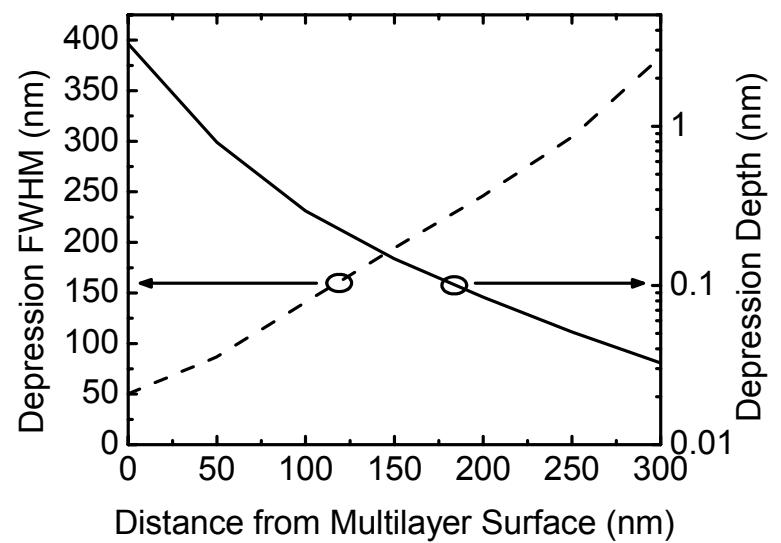

Figure 12 


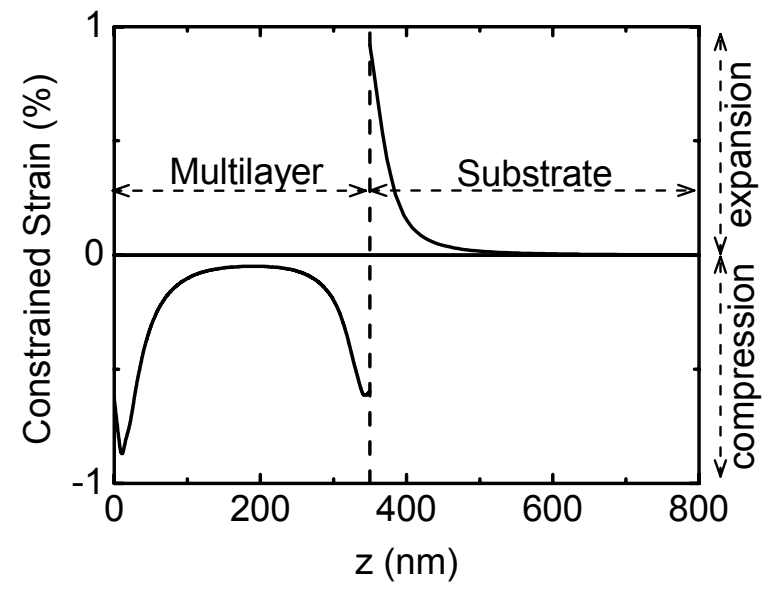

Figure 13

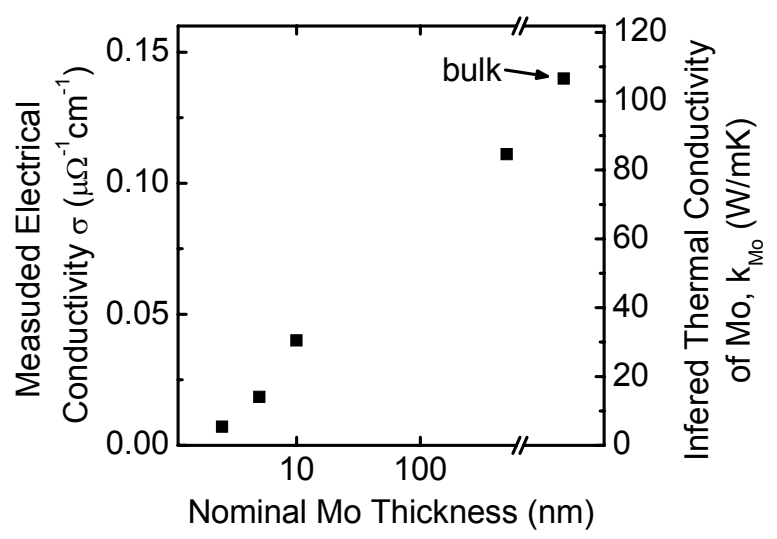

Figure 14 